\title{
On Multicomponent Derivative Nonlinear Schrodinger Equation Related to Symmetric Spaces
}

Tihomir Valchev

Technological University Dublin, Tihomir.Valchev@tudublin.ie

Follow this and additional works at: https://arrow.tudublin.ie/scschmatcon

Part of the Partial Differential Equations Commons

\section{Recommended Citation}

Valchev, T. (2012). On Multicomponent Derivative Nonlinear Schrodinger Equation Related to Symmetric Spaces. Proceedings of the XIV-th International Conference on Geometry, Integrability and Quantization, June 8-13, 2012, Varna, Bulgaria Ivaïlo M. Mladenov, pp 215-226 doi:10.7546/giq-14-2013-215-226

This Conference Paper is brought to you for free and open access by the School of Mathematics at ARROW@TU Dublin. It has been accepted for inclusion in Conference papers by an authorized administrator of ARROW@TU Dublin. For more information, please contact arrow.admin@tudublin.ie, aisling.coyne@tudublin.ie,gerard.connolly@tudublin.ie. Funder: Government of Ireland Postdoctoral Fellowship in Science, Engineering and Technology

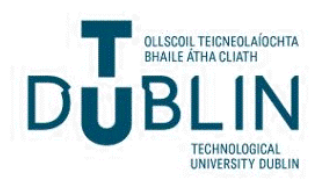




\title{
ON MULTICOMPONENT DERIVATIVE NONLINEAR SCHRÖDINGER EQUATION RELATED TO SYMMETRIC SPACES
}

\author{
TIHOMIR I. VALCHEV
}

Institute for Nuclear Research and Nuclear Energy, Bulgarian Academy of Sciences, 1784 Sofia, Bulgaria

\begin{abstract}
We study derivative nonlinear Schrödinger equations related to symmetric spaces of the type A.III. We discuss the spectral properties of the corresponding Lax operator and develop the direct scattering problem connected to it. By applying an appropriately chosen dressing factor we derive soliton solutions to the nonlinear equation. We find the integrals of motion by using the method of diagonalization of Lax pair.
\end{abstract}

\section{Introduction}

A classical example of a nonlinear evolution equation integrable by means of the inverse scattering transform is provided by derivative nonlinear Schrödinger equation (DNSE)

$$
\mathrm{i} q_{t}+q_{x x}+\mathrm{i}\left(|q|^{2} q\right)_{x}=0,
$$

where function $q: \mathbb{R}^{2} \rightarrow \mathbb{C}$ is infinitely smooth. DNSE occurs in plasma physics to describe the propagation of nonlinear Alfvén waves with circular polarization $[16,17]$. Equation (1.1) is equivalent to compatibility condition $[L, A]=0$ for $L$ and $A$ chosen in the form [13]:

$$
\begin{aligned}
& L(\lambda):=\mathrm{i} \partial_{x}+\lambda Q(x, t)-\lambda^{2} \sigma_{3} \\
& A(\lambda):=\mathrm{i} \partial_{t}+\sum_{k=1}^{3} A_{k}(x, t) \lambda^{k}-2 \lambda^{4} \sigma_{3},
\end{aligned}
$$

where $\lambda \in \mathbb{C}$ is a spectral parameter and

$$
Q(x, t)=\left(\begin{array}{cc}
0 & q(x, t) \\
q^{*}(x, t) & 0
\end{array}\right), \quad \sigma_{3}=\left(\begin{array}{cc}
1 & 0 \\
0 & -1
\end{array}\right) .
$$


The former Lax operator depends quadratically on $\lambda$ and this is why it is said that DNSE is connected to a quadratic bundle.

Since the time the integrability of DNSE was discovered many attempts to generalize it have been made. One possible direction is to consider a quadratic bundle of a general form [7], namely:

$$
L(\lambda):=\mathrm{i} \partial_{x}+U_{0}(x, t)+\frac{1}{2} q_{1} p_{1} \sigma_{3}+\lambda U_{1}(x, t)-\lambda^{2} \sigma_{3},
$$

where

$$
U_{0,1}(x, t)=\left(\begin{array}{cc}
0 & q_{0,1}(x, t) \\
p_{0,1}(x, t) & 0
\end{array}\right) .
$$

A certain reduction of the nonlinear evolution equation related to (1.3) is given by the celebrated Gerdjikov-Ivanov equation:

$$
\mathrm{i} q_{t}+q_{x x}+\mathrm{i} q^{2} q_{x}^{*}+\frac{1}{2}|q|^{4} q=0 .
$$

Another trend of current interest $[8,9,12]$ in theory of integrable systems was pioneered by A. Fordy et al. $[1,3,4]$ who related Lax pairs to different Hermitian symmetric spaces in a very natural geometric way. In [3] Fordy managed to derive relatively simple multicomponent versions of DNSE like the following one:

$$
\mathrm{i} \mathbf{q}_{t}+\mathbf{q}_{x x}+\frac{2 \mathrm{i}}{n+1}\left(\left(\mathbf{q}^{T} \mathbf{q}^{*}\right) \mathbf{q}\right)_{x}=0
$$

where $\mathbf{q}: \mathbb{R}^{2} \rightarrow \mathbb{C}^{n}$ is an infinitely smooth function. Our aim here is to study certain basic properties of equation (1.4) and its Lax pair.

The report is organised as follows. Second section is preliminary in its nature. We discuss some basic properties of the scattering operator $L$ and the linear problem $L \psi=0$ related to the nonlinear evolution equation (1.4) to be used further in text. Next two sections contain our main results. In section 3 we apply dressing method to quadratic bundles in order to generate special types of solutions. In particular, we demonstrate how one can obtain the multisoliton solutions of DNSE. Section 4 is dedicated to the foundations of Hamiltonian formalism. We derive the integrals of motion and introduce a Poisson bracket. For that purpose we make use of a method proposed by Drinfel'd and Sokolov [2]. In section 5 we summarize our results and make some additional remarks.

\section{Quadratic Bundles Related to Hermitian Symmetric Spaces}

In this section we are going to expose very briefly the scattering theory for the Lax operator $L$ related to DNSE (1.4). For this to be done we are going to follow some well-known ideas in soliton theory, see $[10,18]$ for detailed explanations. 
Equation (1.4) represents compatibility condition of the following Lax operators:

$$
\begin{aligned}
& L(\lambda):=\mathrm{i} \partial_{x}+\lambda Q(x, t)-\lambda^{2} J \\
& A(\lambda):=\mathrm{i} \partial_{t}+\sum_{k=1}^{4} \lambda^{k} A_{k}(x, t),
\end{aligned}
$$

where $\lambda \in \mathbb{C}$ is spectral parameter. All coefficients above are assumed to be Hermitian traceless $(n+1) \times(n+1)$ matrices. This requirement can also be viewed $[14,15]$ as $\mathbb{Z}_{2}$ reduction condition

$$
L^{\dagger}\left(\lambda^{*}\right)=\tilde{L}(\lambda), \quad A^{\dagger}\left(\lambda^{*}\right)=\tilde{A}(\lambda)
$$

imposed on a generic $L-A$ pair. Above we have introduced the auxiliary notation $\tilde{L}(\lambda) \psi:=-\mathrm{i} \partial_{x} \psi+\lambda \psi(Q-\lambda J)$. Apart of (2.3) the Lax operators $L$ and $A$ is a subject to the following $\mathbb{Z}_{2}$ reduction:

$$
\mathbf{C} L(-\lambda) \mathbf{C}=L(\lambda), \quad \mathbf{C} A(-\lambda) \mathbf{C}=A(\lambda)
$$

where $\mathbf{C}=\operatorname{diag}(1,-1 \ldots,-1)$. Due to the form of $\mathbf{C}$ the potential $Q$ has the block structure:

$$
Q(x, t)=\left(\begin{array}{cc}
0 & \mathbf{q}^{T}(x, t) \\
\mathbf{q}^{*}(x, t) & 0
\end{array}\right)
$$

while the constant matrix $J$ is block diagonal.

The matrix $\mathbf{C}$ represents action of Cartan's involutive automorphism to define symmetric space $S U(n+1) / S(U(1) \times U(n))$ which is a special case of A.III type symmetric space according to Cartan's classification, see [11] for more details. Cartan's involution induces a $\mathbb{Z}_{2}$ grading in the corresponding Lie algebra $\mathfrak{s l}(n+1)$ (as well as its real compact form $\mathfrak{s u}(n+1)$ ) as follows:

$$
\mathfrak{s l}(n+1)=\mathfrak{s l}^{0}(n+1)+\mathfrak{s l}^{1}(n+1),
$$

where

$$
\mathfrak{s l}^{\sigma}(n+1):=\left\{X \in \mathfrak{s l}(n+1) \mid \mathbf{C} X \mathbf{C}^{-1}=(-1)^{\sigma} X\right\} .
$$

It is easy to see that $Q$ as well as $A_{1}$ and $A_{3}$ belong to $\mathfrak{s l}^{1}(n+1)$ while $J, A_{2}$ and $A_{4}$ belong to $\mathfrak{s l}^{0}(n+1)$. The subspace $\mathfrak{s l}^{0}(n+1)$ consists of all block diagonal traceless matrices. For the sake of convenience we pick up $J=\operatorname{diag}(n,-1, \ldots,-1)$. Thus $\mathfrak{s l}^{0}(n+1)$ coincides with the centralizer of $J$.

In order to get definite results one must impose certain additional boundary conditions on q. We are going to restrict ourselves with the simplest case of zero boundary conditions, i.e. we have:

$$
\lim _{x \rightarrow \pm \infty} \mathbf{q}(x, t)=\mathbf{0}
$$

For such boundary conditions the continuous part of the spectrum of $L$ fills up the real and the imaginary axis in the complex $\lambda$-plane. Indeed, the continuous spectrum of $L$ is a locus of points in $\mathbb{C}$ determined where the exponent $\exp \left(\mathrm{i} \lambda^{2} J x\right)$ 
oscillates $[5,10]$ or equivalently condition $\operatorname{im} \lambda^{2}=0$ holds true ${ }^{1}$. On the other hand due to reductions (2.3) and (2.4) the discrete eigenvalues of $L$ get correlated ${ }^{2}$, namely they go in quadruples $\left\{ \pm \mu_{k}, \pm \mu_{k}^{*}\right\}, k=1, \ldots, n$.

In order for one to formulate direct scattering theory it is necessary to consider auxilary linear problem:

$$
L(\lambda) \psi(x, t, \lambda)=0 .
$$

The function $\psi$ is viewed as a fundamental set of solutions to (2.7) called fundamental solution for short, i.e. $\psi$ takes values in the Lie group $\mathrm{SU}(n+1)$. Since $L$ and $A$ commute any fundamental solution $\psi$ satisfies

$$
A(\lambda) \psi(x, t, \lambda)=\psi(x, t, \lambda) f(\lambda)
$$

as well. The quantity

$$
f(\lambda)=\lim _{x \rightarrow \pm \infty} \sum_{k=1}^{4} \lambda^{k} A_{k}(x, t)=-(n+1) \lambda^{4} J
$$

is called dispersion law. The dispersion law labels the nonlinear evolution equation within the integrable hierarchy ${ }^{3}$ and thus it is a fundamental feature of integrable equations.

Next one introduces Jost solutions through the following equality:

$$
\lim _{x \rightarrow \pm \infty} \psi_{ \pm}(x, t, \lambda) \mathrm{e}^{\mathrm{i} \lambda^{2} J x}=\mathbb{1} .
$$

The Jost solutions are defined only on continuous spectrum of $L(\lambda)$, that is the real and imaginery axes in the $\lambda$-plane.

Any two fundamental solutions are linearly related. The transition matrix

$$
T(t, \lambda)=\psi_{+}(x, t, \lambda)^{-1} \psi_{-}(x, t, \lambda)
$$

between the Jost solutions is called scattering matrix. Its time evolution is driven by the dispersion law as follows:

$$
\mathrm{i} \partial_{x} T+[f(\lambda), T]=0 \quad \Rightarrow \quad T(t, \lambda)=\mathrm{e}^{\mathrm{i} f(\lambda) t} T(0, \lambda) \mathrm{e}^{-\mathrm{i} f(\lambda) t} .
$$

Equation (2.11) represents a linearization of the nonlinear equation under consideration. This crucial fact underlies the interpretation of the inverse scattering method as a nonlinear Fourier transform $[10,18]$.

\footnotetext{
${ }^{1}$ Strictly speaking the spectrum of $L$ is complementary to the domain of its resolvent. More detailed analysis $[5,10]$ shows that this is equivalent to satisfaction of above mentioned condition

${ }^{2}$ All eigenvalues of $L$ must belong to a certain discrete orbit of the reduction group $\mathbb{Z}_{2} \times \mathbb{Z}_{2}[10,14$, 15].

${ }^{3}$ The integrable hierarchy consists of all equations to share the same Lax operator $L$. So they are distinguished by the form of second operator $A$.
} 


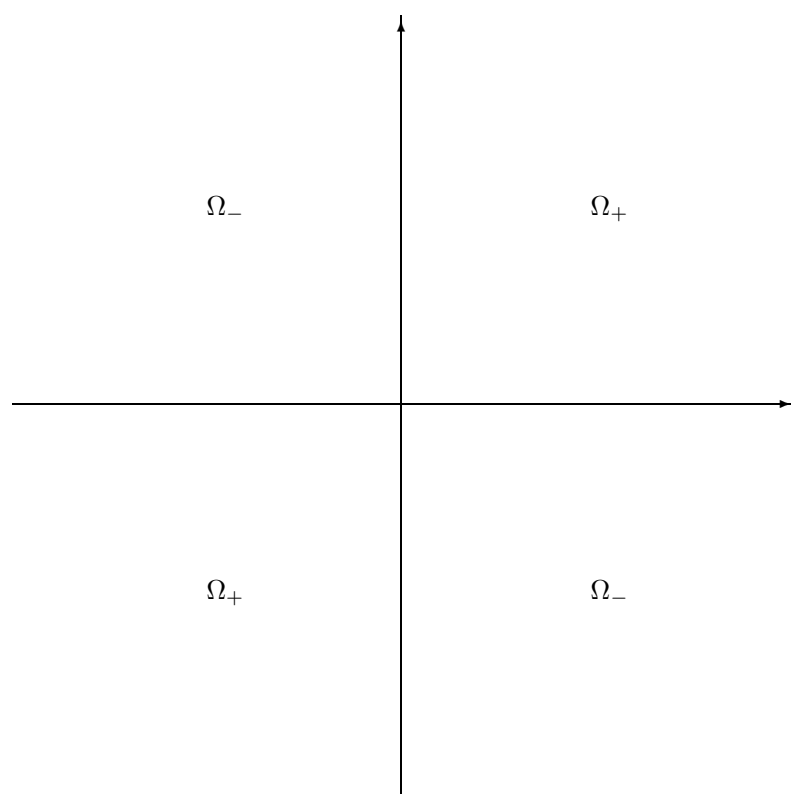

Figure 1. Domains of analyticity.

Similarly to the scalar DNSE $[6,13]$ the Jost solutions to $(2.7)$ can be used to construct in a purely algorithmic way another pair of solutions $\chi^{+}(x, \lambda)$ and $\chi^{-}(x, \lambda)$ that are analytic in domains $\Omega^{+}$and $\Omega^{-}$(see Fig.1). The explicit formulae read:

$$
\chi^{ \pm}(x, \lambda)=\psi_{-}(x, \lambda) S^{ \pm}(\lambda)=\psi_{+}(x, \lambda) T^{\mp}(\lambda) D^{ \pm}(\lambda) .
$$

$S^{+}(\lambda)$ and $T^{+}(\lambda)$ are upper block triangular matrices, $S^{-}(\lambda)$ and $T^{-}(\lambda)$ are lower block triangular matrices, while $D^{ \pm}(\lambda)$ are block diagonal. All these appear in the generalized Gauss decomposition

$$
T(\lambda)=T^{\mp}(\lambda) D^{ \pm}(\lambda)\left(S^{ \pm}(\lambda)\right)^{-1}
$$

of the scattering matrix $T$. It is seen that

$$
\chi^{+}(x, \lambda)=\chi^{-}(x, \lambda) G(\lambda) \quad \lambda \in \mathbb{R} \cup \mathrm{i} \mathbb{R}
$$

for some sewing function $G(\lambda)=\left(S^{-}(\lambda)\right)^{-1} S^{+}(\lambda)$. Thus the fundamental solutions $\chi^{+}(x, \lambda)$ and $\chi^{-}(x, \lambda)$ solve a local Riemann-Hilbert problem defined on the continuous spectrum of $L$. The reductions (2.3) and (2.4) impose certain symmetry conditions on the Jost solutions, the scattering matrix and fundamental analytic 
solutions. Here is a list of these:

$$
\begin{array}{rlr}
{\left[\psi_{ \pm}^{\dagger}\left(x, \lambda^{*}\right)\right]^{-1}} & =\psi_{ \pm}(x, \lambda) & {\left[T^{\dagger}\left(\lambda^{*}\right)\right]^{-1}=T(\lambda)} \\
\mathbf{C} \psi_{ \pm}(x,-\lambda) \mathbf{C} & =\psi_{ \pm}(x, \lambda) & \mathbf{C} T(-\lambda) \mathbf{C}=T(\lambda) \\
{\left[\chi^{+}\left(x, \lambda^{*}\right)\right]^{\dagger}} & =\left[\chi^{-}(x, \lambda)\right]^{-1} & \mathbf{C} \chi^{+}(x,-\lambda) \mathbf{C}=\chi^{-}(x, \lambda) .
\end{array}
$$

\section{Dressing method and special solutions}

In this section we are going to present an algorithm to obtain particular solutions to DNSE. This algorithm is based on Zakharov-Shabat dressing technique [18, 19] adapted for quadratic bundles. This very effective method allows one to derive in an algebraic manner the soliton solutions to DNSE.

\subsection{Dressing method}

We shall sketch here very briefly the concept of the dressing method (DM). DM is an indirect method for integration, i.e. it uses a known (simple) solution to a given equation to produce another (more complicated) one. For this to be done it takes into account the existence of Lax representation.

Let $\psi_{0}$ be a fundamental solution to

$$
L_{0} \psi_{0}=\mathrm{i} \partial_{x} \psi_{0}+\lambda\left(Q_{0}-\lambda J\right) \psi_{0}=0 .
$$

where

$$
Q_{0}(x)=\left(\begin{array}{cc}
0 & \mathbf{q}_{0}(x) \\
\mathbf{q}_{0}^{*}(x) & 0
\end{array}\right)
$$

for some vector $\mathbf{q}_{0}^{T}=\left(q_{0}^{1}, \ldots, q_{0}^{n}\right)$ assumed to be a known solution to the nonlinear equation. Then we construct another function $\psi_{1}(x, \lambda):=g(x, \lambda) \psi_{0}(x, \lambda)$ by "dressing" the initial solution $\psi_{0}$. Assuming that $\psi_{1}$ satisfies the linear problem

$$
L_{1} \psi_{1}=\mathrm{i} \partial_{x} \psi_{1}+\lambda\left(Q_{1}-\lambda J\right) \psi_{1}=0
$$

defined for some potential

$$
Q_{1}(x):=\left(\begin{array}{cc}
0 & \mathbf{q}_{1}(x) \\
\mathbf{q}_{1}^{*}(x) & 0
\end{array}\right)
$$

to be found. Comparing (3.1) and (3.2) we see that the dressing factor $g$ satisfies:

$$
\mathrm{i} \partial_{x} g+\lambda Q_{1} g-\lambda g Q_{0}-\lambda^{2}[J, g]=0 .
$$

The $\mathbb{Z}_{2}$ reductions (2.12)-(2.14) imply that $g$ fulfills a similar set of symmetry conditions, namely:

$$
\begin{aligned}
{\left[g^{\dagger}\left(x, \lambda^{*}\right)\right]^{-1} } & =g(x, \lambda) \\
\mathbf{C} g(x,-\lambda) \mathbf{C} & =g(x, \lambda) .
\end{aligned}
$$


The simplest nontrivial choice for $g$ to meet these requirements reads:

$$
g(x, \lambda)=\mathbb{1}+\frac{\lambda B(x)}{\mu(\lambda-\mu)}+\frac{\lambda \mathbf{C} B(x) \mathbf{C}}{\mu(\lambda+\mu)}, \quad \Re \mu \neq 0, \Im \mu \neq 0
$$

From (3.3) it follows that $Q_{1}$ and $Q_{0}$ are interrelated through:

$$
Q_{1}=A Q_{0} A^{\dagger}+[J, B-\mathbf{C} B \mathbf{C}] A^{\dagger},
$$

where

$$
A=\mathbb{1}+\frac{1}{\mu}(B+\mathbf{C} B \mathbf{C}) .
$$

Thus we have expressed $Q_{1}$ in terms of the seed solution $Q_{0}$ and the residue $B$. In order to find $B$ one analyzes the identity $g g^{-1}=11$. After calculating the residue at $\lambda=\mu$ we obtain algebraic relation

$$
B\left(\mathbb{1}+\frac{\mu B^{\dagger}}{\mu^{*}\left(\mu-\mu^{*}\right)}+\frac{\mu \mathbf{C} B^{\dagger} \mathbf{C}}{\mu^{*}\left(\mu+\mu^{*}\right)}\right)=0 .
$$

$B(x, t)$ must be a degenerate matrix. ${ }^{4}$ Therefore there exist rectangular $(n+1) \times k$ matrices $X(x)$ and $F(x)$ to fulfill $B=X F^{T}$. Then (3.7) is reduced to an algebraic equation for $X$ that can be solved easily to give:

$$
X=\frac{\mu}{\mu^{*}}\left(\frac{F^{T} F^{*}}{\mu-\mu^{*}}-\frac{F^{T} \mathbf{C} F^{*}}{\mu+\mu^{*}} \mathbf{C}\right)^{-1} F^{*} .
$$

The factor $F$ can be found from differential equation (3.3). Detailed analysis shows that it is expressed through:

$$
F^{T}(x)=F_{0}^{T}\left[\psi_{0}(x, \mu)\right]^{-1},
$$

where $\psi_{0}$ is any fundamental solution to (3.1) defined at a vicinity of $\mu$ and $F_{0}$ is a constant matrix.

What remains is to recover the time evolution. For this to be done one must consider equation

$$
\mathrm{i} \partial_{t} g+\sum_{k=1}^{2 N} \lambda^{k} A_{k}^{(1)} g-g \sum_{k=1}^{2 N} \lambda^{k} A_{k}^{(0)}=0
$$

that follows from the second linear problem (2.8). Skipping all details we present the final result: the matrix $F_{0}$ evolves with time according to

$$
\mathrm{i} \partial_{t} F_{0}^{T}-F_{0}^{T} f(\mu)=0 .
$$

Thus we are able to propose a simple rule to derive the time dependence of $Q_{1}$, namely:

$$
F_{0}^{T} \rightarrow F_{0}^{T} \mathrm{e}^{-\mathrm{i} f(\mu) t}
$$

\footnotetext{
${ }^{4}$ If $B$ is invertible then from (3.7) we see that it is proportional to $\mathbb{1 1}$, that is we have trivial dressing.
} 


\subsection{Soliton solutions}

The simplest class of solutions are solitons. To derive 1-soliton solution we set

$$
Q_{0}=0 \quad \Rightarrow \quad \psi_{0}(x, t, \lambda)=\mathrm{e}^{-\mathrm{i} \lambda^{2} J x} .
$$

We shall resrict ourselves here with the case when $\operatorname{rank} B=1$. Then the reflectionless potential acquires the form:

$$
\begin{aligned}
q_{1}^{j-1}(x)= & \left(Q_{1}\right)_{1 j}(x)=2 \mathrm{i}(n+1) \sum_{l=2}^{n+1} \frac{\rho \sin (2 \varphi) \mathrm{e}^{-\mathrm{i} \sigma_{l}(x)} \mathrm{e}^{\theta_{l}(x)}}{\mathrm{e}^{-2 \mathrm{i} \varphi}+\sum_{p=2}^{n+1} \mathrm{e}^{2 \theta_{p}(x)}} \times \\
& \left(\delta_{j l}-2 \mathrm{i} \sin (2 \varphi) \frac{\mathrm{e}^{\theta_{j}(x)+\theta_{l}(x)} \mathrm{e}^{\mathrm{i}\left(\delta_{j}-\delta_{l}-2 \varphi\right)}}{\mathrm{e}^{-2 \mathrm{i} \varphi}+\sum_{p=2}^{n+1} \mathrm{e}^{2 \theta_{p}(x)}}\right),
\end{aligned}
$$

where we have used the notation:

$$
\begin{array}{rlrl}
\theta_{p}(x) & =(n+1) \rho^{2} \sin (2 \varphi) x-\xi_{0, p} & p=2, \ldots, n+1 \\
\sigma_{p}(x) & =(n+1) \cos (2 \varphi) x+\delta_{1}-\delta_{p}-\varphi & \mu=\rho \exp (\mathrm{i} \varphi) \\
\xi_{0, p} & =\ln \left|F_{0,1} / F_{0, p}\right| \quad \delta_{1}=\arg F_{0,1} & \delta_{p}=\arg F_{0, p} .
\end{array}
$$

In order to obtain the 1-soliton solution we need to recover the time dependence in (3.12). For that purpose one should use (3.11) and take into account that for the DNSE $f(\lambda)=-(n+1) \lambda^{4} J$. As a result one gets the following correspondence:

$$
\begin{aligned}
\xi_{0, p} & \rightarrow \xi_{0, p}-2(n+1) \rho^{4} \sin (4 \varphi) t \\
\delta_{1} & \rightarrow \delta_{1}+2 n \rho^{4} \cos (4 \varphi) t, \quad \delta_{p} \rightarrow \delta_{p}-2 \rho^{4} \cos (4 \varphi) t .
\end{aligned}
$$

It is clear that one is able to apply the dressing procedure to the 1-soliton solution and thus constructs a 2 -soliton solution and so on. This way one obtains a sequence of exact solutions. There exists another approach in deriving the multisoliton solutions however. It consists in using a multiple pole dressing factor of the form:

$$
g(x, t, \lambda)=\mathbb{1}+\sum_{k=1}^{N} \frac{\lambda}{\mu_{k}}\left(\frac{B_{k}(x, t)}{\lambda-\mu_{k}}+\frac{\mathbf{C} B_{k}(x, t) \mathbf{C}}{\lambda+\mu_{k}}\right),
$$

where all $\mu_{k}$ do not lie on the continuous spectrum of $L$. Then the multisoliton solution is obtained through the following formula:

$$
Q_{1}=\sum_{k=1}^{m}\left[J, B_{k}-\mathbf{C} B_{k} \mathbf{C}\right] A^{\dagger},
$$

where

$$
A=\mathbb{1}+\sum_{k=1}^{m} \frac{1}{\mu_{k}}\left(B_{k}+\mathbf{C} B_{k} \mathbf{C}\right) .
$$


To find the residues $B_{k}=X_{k} F_{k}^{T}$ one follows basically the same steps as in the two poles case. The matrices $F_{k}$ are determined by the value of some bare solution at the corresponding pole $\mu_{k}$ :

$$
F_{k}^{T}(x, t)=F_{k, 0}^{T}\left[\psi_{0}\left(x, t, \mu_{k}\right)\right]^{-1}
$$

while $X_{k}$ can be found by solving the linear system:

$$
F_{k}^{*}=\sum_{l=1}^{m} \frac{\mu_{k}^{*}}{\mu_{l}}\left(X_{l} \frac{F_{l}^{T} F_{k}^{*}}{\mu_{l}-\mu_{k}^{*}}-\mathbf{C} X_{l} \frac{F_{l} \mid \mathbf{C} F_{k}^{*}}{\mu_{l}+\mu_{k}^{*}}\right) .
$$

Finally the time dependence is recovered through the rule:

$$
F_{k, 0}^{T} \rightarrow F_{k, 0}^{T} \mathrm{e}^{-\mathrm{i} f\left(\mu_{k}\right) t}
$$

to generalize (3.11) in a very natural way.

\section{Integrals of Motion}

In this section we develop some basics of the Hamiltonian formulation of DNSE related to symmetric spaces. Firstly we are going to describe (local in $x$ and $t$ ) conserved densities by deriving a general recursive formula to generate them. In order to do this we shall use the method of diagonalization of Lax pair proposed by Drinfel'd and Sokolov [2].

Let us consider the following general flow Lax pair:

$$
\begin{aligned}
& L(\lambda):=\mathrm{i} \partial_{x}+\lambda Q(x, t)-\lambda^{2} J \\
& A(\lambda):=\mathrm{i} \partial_{t}+\sum_{k=1}^{2 N} A_{k}(x, t) \lambda^{k}
\end{aligned}
$$

After applying a gauge transform

$$
\mathcal{P}(x, t, \lambda)=\mathbb{1}+\sum_{k=1}^{\infty} \frac{p_{k}(x, t)}{\lambda^{k}}, \quad p_{k} \in \mathfrak{s l}^{1}(n+1)
$$

one can put the $L-A$ pair into diagonal form:

$$
\begin{aligned}
& \mathcal{L}=\mathcal{P}^{-1} L \mathcal{P}=\mathrm{i} \partial_{x}-\lambda^{2} J+\lambda \mathcal{L}_{-1}+\mathcal{L}_{0}+\frac{\mathcal{L}_{1}}{\lambda}+\cdots \\
& \mathcal{A}=\mathcal{P}^{-1} A \mathcal{P}=\mathrm{i} \partial_{t}+\sum_{k=1}^{2 N} \lambda^{k} \mathcal{A}_{-k}+\mathcal{A}_{0}+\frac{\mathcal{A}_{1}}{\lambda}+\cdots
\end{aligned}
$$


that is all coefficients above are elements of $\mathfrak{s l}^{0}(n+1)$. Since the zero curvature representation is invariant under gauge transforms we have:

$$
\partial_{t} \mathcal{L}_{k}-\partial_{x} \mathcal{A}_{k}+\sum_{l}^{k}\left[\mathcal{L}_{l}, \mathcal{A}_{k-l}\right]=0 .
$$

From (4.6) it is seen that the matrix element $\left(\mathcal{L}_{k}\right)_{11}$ or equivalently the trace of the $n \times n$ block of $\mathcal{L}_{k}$ represent local densities of integrals of motion. To find these densities one substitutes (4.3) into (4.4) and then compares coefficitents before the same powers of $\lambda$. As a result one obtains the following set of recurrence relations:

$$
\begin{aligned}
\lambda & : \mathcal{L}_{-1}-p_{1} J=Q-J p_{1}, \\
\lambda^{0} & : \mathcal{L}_{0}+p_{1} \mathcal{L}_{-1}-p_{2} J=Q p_{1}-J p_{2}, \\
\lambda^{-k} & : \mathcal{L}_{k}+\sum_{l=1}^{k+1} p_{l} \mathcal{L}_{k-l}=\mathrm{i} p_{k, x}+Q p_{k+1}-\left[J, p_{k+2}\right], \quad k=1,2, \ldots
\end{aligned}
$$

After projecting the first two recurrence relations into a block diagonal and offblock diagonal part we deduce that:

$$
\begin{aligned}
\mathcal{L}_{-1} & =0, \quad p_{1}=\operatorname{ad}_{J}^{-1} Q=\frac{1}{n+1}\left(\begin{array}{cc}
0 & \mathbf{q}^{T} \\
-\mathbf{q}^{*} & 0
\end{array}\right) \\
\mathcal{L}_{0} & =Q p_{1}=\frac{1}{n+1}\left(\begin{array}{cc}
-\mathbf{q}^{T} \mathbf{q}^{*} & 0 \\
0 & \mathbf{q}^{*} \mathbf{q}^{T}
\end{array}\right), \quad p_{2}=0 .
\end{aligned}
$$

Thus the first integral density is $I_{1}=\mathbf{q}^{\dagger} \mathbf{q}$. The results just obtained are generalized in the following theorem to be given here without proof:

Theorem 1. All conserved densities $\mathcal{L}_{k}$ corresponding to odd indices vanish while the rest are generated by the following formula:

$$
\mathcal{L}_{k}=Q p_{k+1},
$$

where the matrix coefficient $p_{k}$ can be found through the recursive formula:

$$
p_{k}=\operatorname{ad}_{J}^{-1}\left(\mathrm{i} p_{k-2, x}-\sum_{l=1}^{k-2} p_{l} \mathcal{L}_{k-2-l}\right) . \square
$$

Taking into account the statement of the theorem it is easily seen that next nonzero integral density reads:

$$
I_{2}=\mathrm{iq}^{\dagger} \mathbf{q}_{x}-\frac{1}{n+1}\left(\mathbf{q}^{\dagger} \mathbf{q}\right)^{2}
$$

It represents the Hamiltonian density $H$ of the multicomponent DNLS equation if we choose Poisson bracket as follows:

$$
\{F, G\}:=\int_{-\infty}^{\infty} \mathrm{d} y \operatorname{tr}\left(\frac{\delta F}{\delta Q} \partial_{x} \frac{\delta G}{\delta Q^{T}}\right) .
$$




\section{Conclusions}

The direct scattering problem for quadratic bundle related to Hermitian symmetric spaces of the type A.III has been formulated and discussed. We have suited the Zakharov-Shabat dressing technique to quadratic bundles related to Hermitian symmetric spaces of the mentioned type. This allowed us to construct analytically the multisoliton solutions. In particular, the one soliton solution has been written down explicitly. This result naturally generalizes the classical result by Kaup and Newell [13] for the soliton solution to scalar DNSE. The latter can be derived by using a dressing factor chosen in the form:

$$
g(x, \lambda)=\mathbb{1}+\frac{\lambda B}{\mu(\lambda-\mu)}+\frac{\lambda \sigma_{3} B \sigma_{3}}{\mu(\lambda+\mu)} .
$$

We have described the integrals of motion for the DNSE (1.4) by deriving a general recursion formula. In order to do this we have applied the method of diagonalization of Lax pair. As a simple illustration we have calculated the first two integrals of motion. The latter represents the Hamiltonian of DNSE when the Poisson bracket is defined as in (4.15). All this underlies the Hamiltonian formalism for the corresponding nonlinear equation.

All results presented in this report can be extended in several directions. Firstly, one can consider quadratic bundles (2.1) related to other Hermitian symmetric spaces, say BD.I type symmetric spaces. Since the corresponding DNSE look more complicated way than (1.4) so the theory of such equations would be more complicated than in A.III case.

Another meaningful direction consists in studying complete quadratic bundles, i.e. Lax operators in the form:

$$
L(\lambda)=\mathrm{i} \partial_{x}+Q_{0}+\lambda Q_{1}-\lambda^{2} J,
$$

where $Q_{0}$ splits into a diagonal and off-diagonal part, $Q_{1}$ is strictly off-diagonal and $J$ is a diagonal matrix. It is evident that the theory of complete quadratic bundles gets more complicated than the one related to symmetric spaces.

\section{Acknowledgements}

The author would like to thank Prof. V. Gerdjikov and Dr. R. Ivanov for fruitful discussions and support.

\section{References}

[1] Athorne C. and Fordy A., Generalised KdV and MKdV Equations Associated with Symmetric Spaces, J. Phys. A 20 (1987) 1377-1386. 
[2] Drinfel'd V. and Sokolov V., Lie Algebras and Equations of Korteweg-de Vries Type, Sov. J. Math. 30 (1985) 1975-2036.

[3] Fordy A., Derivative Nonlinear Schrödinger Equations and Hermitian Symmetric Spaces, J. Phys. A: Math. Gen. 17 (1984) 1235-1245.

[4] Fordy A. and Kulish P., Nonlinear Schrödinger Equations and Simple Lie Algebras, Commun. Math. Phys. 89 (1983) 427-443.

[5] Gerdjikov V., On Spectral Theory of Lax Operators on Symmetric Spaces: Vanishing Versus Constant Boundary Conditions, JGSP 15 (2009) 1-41.

[6] Gerdjikov V. and Ivanov M., The Quadratic Bundle of General Form and the Nonlinear Evolution Equations. I. Expansions over the "Squared" Solutions - Generalized Fourier Transform, Bulg. J. Phys. 10 (1983) 13-26.

[7] Gerdjikov V. and Ivanov M., The Quadratic Bundle of General Form and the Nonlinear Evolution Equations. II. Hierarchies of Hamiltonian Structures, Bulg. J. Physics 10 (1983) 130-143.

[8] Gerdjikov V., Grahovski G., Mikhailov A. and Valchev T., Polynomial Bundles and Generalised Fourier Transforms for Integrable Equations on A.III-type Symmetric Spaces, SIGMA 7 (2011) 096 (48 pages).

[9] Gerdjikov V., Kostov N. and Valchev T., Solutions of Multi-component NLS Models and Spinor Bose-Einstein Condensates, Physica D 238 (2009) 1306-1310.

[10] Gerdjikov V., Vilasi G. and Yanovski A., Integrable Hamiltonian Hierarchies. Spectral and Geometric Methods, Lecture Notes in Physics $\mathbf{7 4 8}$ Springer Verlag, Berlin, Heidelberg, New York, 2008.

[11] Helgasson S., Differential Geometry, Lie Groups and Symmetric Spaces, Academic Press, New York, 1978.

[12] Ieda J., Miyakawa T. and Wadati M., Exact Analysis of Soliton Dynamics in Spinor Bose-Einstein Condensates, Phys. Rev Lett. 93 (2004) 194102.

[13] Kaup D. and Newell A., An Exact Solution for a Derivative Nonlinear Schrödinger Equation, J. Math. Phys. 19 (1978) 798-801.

[14] Mikhailov A., Reductions in Integrable Systems. The Reduction Groups, Lett. in Jour. of Exper. and Theor. Phys. 32 (1980) 187-192.

[15] Mikhailov A., The Reduction Problem and the Inverse Scattering Method, Physica D 3 (1981) 73-117.

[16] Mio K., Ogino T., Minami K. and Takeda S., Modified Nonlinear Schrödinger Equation for Alfvén Waves Propagating along the Magnetic Field in Cold Plasmas, J. Phys. Soc. Japan 41 (1976) 265-271.

[17] Mjolhus E., On the Modulational Instability of Hydromagnetic Waves Parallel to the Magnetic Field, J. Plasma Physics 16 (1976) 321-334.

[18] Zakharov V. E., Manakov S., Novikov S. and Pitaevskii L., Theory of Solitons: The Inverse Scattering Method, Plenum, New York, (1984).

[19] Zakharov V. and Shabat A., Integration of Nonlinear Equations of Mathematical Physics by the Method of the Inverse Scattering Transform II, Funct. Anal. Appl. 13 (1979) 13-22 (in Russian). 